Editorial de Apresentação da Revista

\title{
OCEANO, HUMANIDADE E REGIÕES TROPICAIS: NOSSO FUTURO DEPENDE DA NOSSA RECONEXÃO
}

OCEAN, HuMANITY AND TROPICAL REGIONS: oUR fUtURE DEPENDS ON OUR RECONNECTION

\section{Luís Felipe Skinner}

1. Universidade do Estado do Rio de Janeiro, Departamento de Ciências, Laboratório de Ecologia e Dinâmica Bêntica Marinha. Rua Francisco Portela, 1470 - Patronato, São Gonçalo RJ, CEP 24435-005. Programa de Pós-graduação em Oceanografia - PPGOCn UERJ

* Autor DE CORRESPONDÊNCIA: LSKINNER@UERJ.BR ORCID: 0000-0003-0971-4870

\begin{tabular}{|l|l|}
\hline RECEBIDO EM 10 DE DEZEMBRO DE 2019 & $\begin{array}{l}\text { CITAÇÃO: } \\
\text { Skinner L.F. OCEANO, HUMANIDADE E }\end{array}$ \\
\hline $\begin{array}{l}\text { VERSÃO REVISADA EM } 10 \text { DE JANEIRO DE } \\
\text { REGIÕES TROPICAIS: NOSSO FUTURO } \\
\text { DEPENDE DA NOSSA RECONEXÃO. J. of } \\
\text { Human and Environment of Tropical } \\
\text { Bays } 1: 1-8,2020 .\end{array}$ \\
\hline ACEITO EM 10 DE JANEIRO DE 2020 & \\
\hline PUBLICADO EM 17 DE JANEIRO DE 2020 & \\
\hline $\begin{array}{l}\text { EDITOR RESPONSÁVEL: M.A. MILWARD-DE- } \\
\text { AZEVEDO (UFRRJ) }\end{array}$ & \\
\hline
\end{tabular}

\section{RESUMO}

Os oceanos cobrem aproximadamente 390 milhões de $\mathrm{km}^{2}$ da superfície da Terra e 66,9 milhões $\mathrm{km}^{2}$ se encontra na região tropical. Os oceanos tropicais abrangem a maior riqueza de espécies e o maior número de relações ecológicas entre estas, em diversos ecossistemas, como recifes de coral e recifes rochosos, manguezais, pradarias de fanerógamas marinhas e praias por exemplo. Ao mesmo tempo, as regiões a até $100 \mathrm{~km}$ da costa são locais de vida para mais de 2,4 bilhões de pessoas, o que corresponde a aproximadamente $40 \%$ da população mundial. Grande parte desta população vive em regiões tropicais e sem acesso à serviços básicos como saneamento ou acesso a água potável tratada. A relação da Humanidade com os oceanos remete há cerca de 164.000 anos no passado, quando o Homem passou a utilizar recursos e serviços marinhos e aprendeu a interpretar e conhecer os ritmos deste ambiente. Este conhecimento foi transmitido de geração a geração e entre diferentes culturas, gerando processos de manejo e uso dos sistemas marinhos de forma sustentável. Nos dias atuais, em que a Humanidade se defronta com a destruição acelerada de habitats e perda de diversidade e inúmeros recursos e serviços ambientais, a redescoberta e a reconexão com o 
ambiente fazem-se extremamente necessários. É neste propósito que a Revista Human and Environment of Tropical Bays se apresenta como um espaço de veiculação de informação de qualidade, que alia o conhecimento sobre o meio físico e biológico dos ecossistemas costeiros tropicais, mas também, da presença do Homem sobre este espaço e de suas ações, visando entender como Humanidade e Ambiente são cada vez mais indissociáveis.

\section{ABSTRACT}

Oceans cover approximately 390 million km² of the Earth's surface and 66.9 million $\mathrm{km}^{2}$ are in the tropical region. Tropical oceans comprise the greatest species richness and the largest number of ecological relationships between them in diverse ecosystems such as coral reefs and rocky reefs, mangroves, seagrasses beds and beaches. At the same time, regions within $100 \mathrm{~km}$ of the coast are living places for over 2.4 billion people, which is approximately $40 \%$ of the world's population. Much of this population lives in tropical regions without access to basic services such as treated sewage or access to safe drinking water. Humanity's relationship with the oceans dates back about 164,000 years ago, when man began to use marine resources and services and learned to interpret and know the rhythms of this environment. This knowledge was transmitted from generation to generation and between different cultures, generating processes of management and use of marine systems in a sustainable manner. Nowadays, when humanity is facing the accelerated destruction of habitats and loss of diversity and numerous environmental resources and services, rediscovery and reconnection with the environment is extremely necessary. It is for this purpose that the Journal of Human and Environment of Tropical Bays presents itself as a space for the dissemination of high quality information, which combines knowledge about the physical and biological environment of tropical coastal ecosystems with the presence of Humans on this space and of their actions, aiming to understand how Humanity and Environment are increasingly inseparable.

Os oceanos cobrem aproximadamente 390 milhões de $\mathrm{km}^{2}$ da superfície da Terra, corresponde a cerca de $72 \%$ da superfície total. Grande parte deste oceano se encontra na região tropical (66,9 milhões $\mathrm{km}^{2}$ ), representando $50 \%$ da área de mar aberto e 30\% da área sobre a plataforma continental (LONGHUSRT e PAULY, 1987). As áreas sob influência tropical, ou seja, com temperatura média acima de $20{ }^{\circ} \mathrm{C}$ estendem-se para além da região compreendida entre os trópicos de Câncer e Capricórnio (Figura 1). 


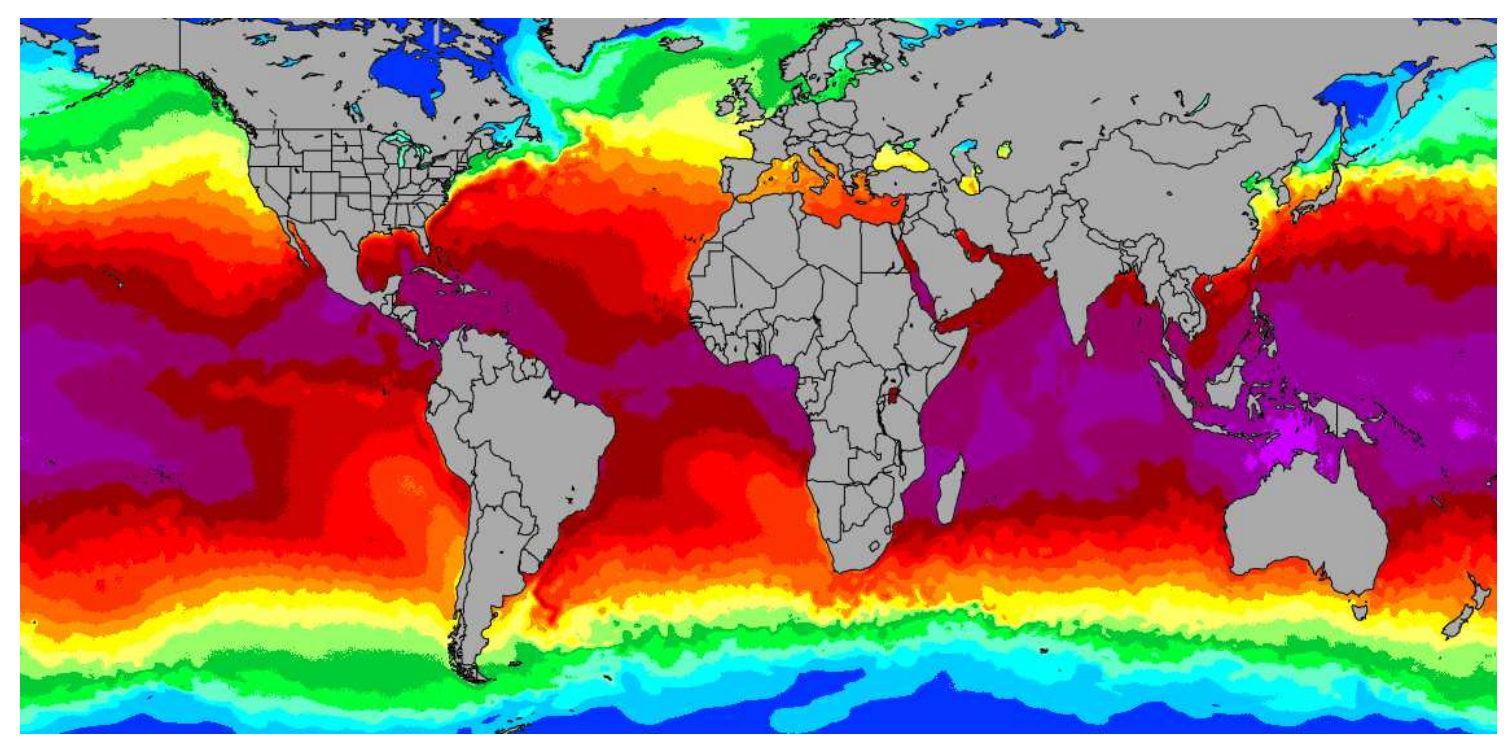

\section{$\begin{array}{lllllllllllllllll}{ }^{\circ} \mathrm{C} & 0 & 1 & 3 & 5 & 7 & 9 & 11 & 13 & 15 & 17 & 19 & 21 & 24 & 27 & 30 & 35\end{array}$}

Figura 1: Mapa apresentando a temperatura superficial da água do mar $\left({ }^{\circ} \mathrm{C}\right)$ nos diferentes oceanos. Fonte: www.seatemperature.org

Os oceanos tropicais abrangem também a maior riqueza de espécies (Figura 2) e o maior número de relações ecológicas entre estas, em diversos ecossistemas, como recifes de coral e recifes rochosos, manguezais, pradarias de fanerógamas marinhas e praias por exemplo (TITTENSOR et al., 2010).

As regiões a até $100 \mathrm{~km}$ da costa são locais de vida para mais de 2,4 bilhões de pessoas, o que corresponde a aproximadamente $40 \%$ da população mundial (UNDP, 2019). Além disso, grande parte desta população vive em regiões tropicais, muitas vezes sem acesso à serviços básicos como acesso à água tratada ou esgotamento sanitário adequado (CIESIN, 2018; SKINNER, 2017) (Figura 3). 


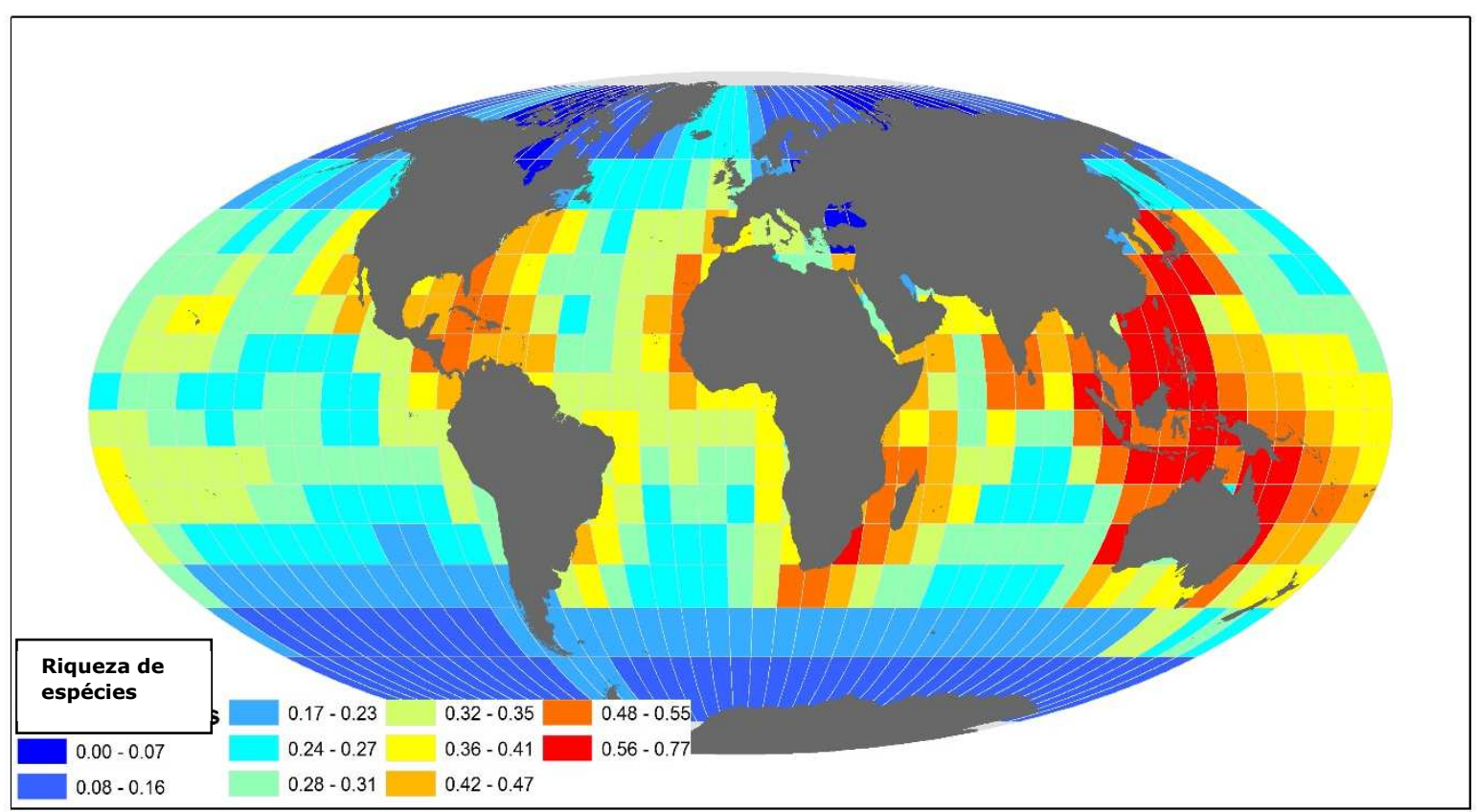

Figura 2: Padrão global de riqueza de espécies nos oceanos. Fonte: Tittensor et al. 2010.

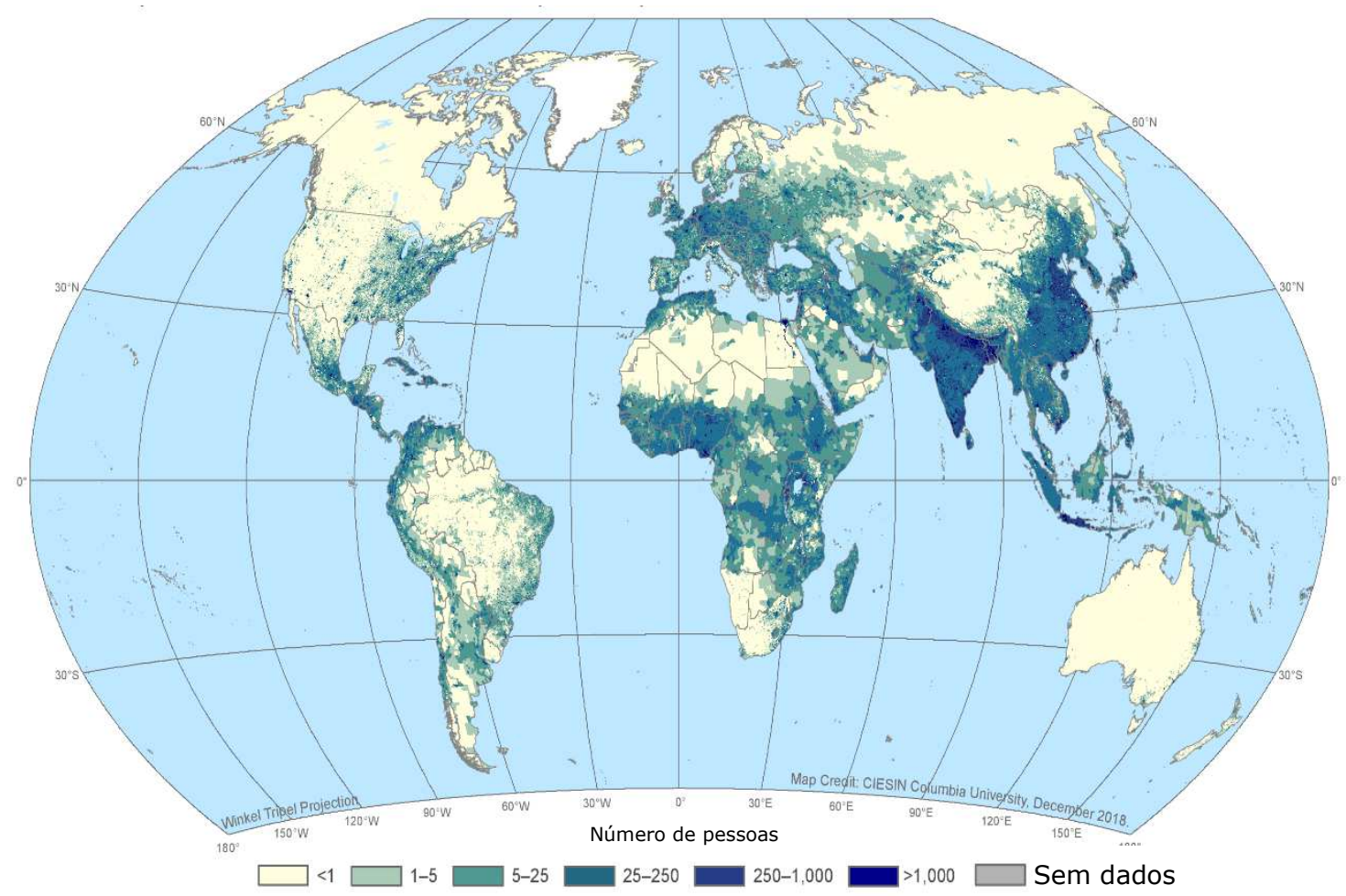

Figura 3: Estimativas da densidade da população mundial para o ano de 2020. Fonte: Center for International Earth Science Information Network - CIESIN - Columbia University. 2018 
A relação da população mundial com as áreas marinhas, sobretudo com as áreas costeiras é enorme, pois dela são extraídos inúmeros produtos e utilizados diversos serviços ambientais. Esta relação da Humanidade com os Oceanos é bastante antiga, com registros que datam desde 164.000 anos antes do presente (AP), quando a espécie Humana ampliou sua dieta e passou a incluir frutos do mar em sua alimentação, no extremo sul do continente Africano (MAREAN et al., 2007), ou há 125.000 anos AP, no entorno do Mar Vermelho. A adaptação aos ambientes costeiros foi uma realidade e pressão evolutiva das populações humanas em expansão, em busca de recursos alimentares, mas também, em novos locais de moradia e caça e em modos de deslocamento mais rápidos e eficientes. Esta interação do ser humano com as zonas costeiras significou o aprendizado de muito dos ciclos biológicos e oceanográficos, como o sobe e desce das marés, ou os sinais de tempestades (MAREAN, 2011). Muitos dos aspectos comportamentais de estratégias de coleta e pesca permanecem sendo utilizadas até os dias atuais, como no caso de muitos moluscos e crustáceos, cuja captura se dá durante os horários e dias de marés mais baixas.

Esta adaptação do conhecimento e exploração dos ambientes costeiros é bastante similar na história dos povos, produzindo estratégias similares de captura e de descarte das partes inservíveis em depósitos dos restos (montes de conchas), indicando um traço comportamental e evolutivo comum. A datação destes depósitos em épocas próximas, como os registros nos Estados Unidos (atual Califórnia) entre 10 e 11.000 anos AP (ERLANDSSON e MOSS, 2001) e os registros brasileiros, há 8.700 anos AP aproximadamente (LOPES et al., 2016) indicam a velocidade e rotas da dispersão humana e também, a riqueza de recursos naturais encontrados e as soluções tecnológicas existentes à época para adaptação ao ambiente. Os depósitos conchíferos são ótimos indicadores da presença humana, e foram utilizados para comprovar a ocupação da Austrália há 2.500 anos AP (FAULKNER, 2011) e da Nova Zelândia, com os Maori, há 800 anos.

Diversos trabalhos mostram a relação entre a expansão das atividades humanas e os impactos nas áreas costeiras adjacentes, relação esta nem sempre harmoniosa, mas cujos efeitos tanto no apogeu das civilizações quanto em seu declínio são nítidos (BRILLES et al., 2019). Em muitas culturas tradicionais, o uso sustentável dos ecossistemas é uma realidade, sendo 
muitas vezes utilizado como modelo à sustentabilidade ambiental e à conservação, incluindo o turismo (BARROS \& RODRIGUES, 2019; SINAY et al., 2019) e o modo de exploração de recursos naturais (TORRE-CASTRO \& RÖNNBÄCK, 2004). Destes exemplos surge também o conceito de SERVIÇOS AO ECOSSISTEMA (COMBERTI et al., 2015), que considera que técnicas tradicionais (indígenas/nativas) de manejo de fauna e flora são importantes para a manutenção da biodiversidade de uma determinada região.

Dam Lam et al. (2019) reuniram informações de 109 artigos publicados e mostraram que a maioria dos estudos (68\%) referem-se à países desenvolvidos, do qual apenas parte dos Estados Unidos e Austrália podem ser inseridos dentro do domínio tropical. Assim, a maior parte dos territórios sob influência tropical é pouco estudado. E uma das questões levantadas para esta carência de estudos é o direito ao uso da terra por povos tradicionais e seu reconhecimento por parte dos governos, o que nem sempre ocorre.

Isto claramente opõe o conhecimento tradicional e a conservação e manejo de espécies e ecossistemas costeiros à destruição destes mesmos estoques pesqueiros e ecossistemas. Manguezais, restingas, recifes de coral, praias e costões rochosos estão entre os ecossistemas marinhos/costeiros mais afetados pelo avanço populacional desordenado e irregular. A destruição destes ecossistemas afeta diretamente a abundância de espécies importantes economicamente ou nutritivamente. Por conseguinte, afetam as populações tradicionais, que ou são deslocadas de seu território em busca de alternativas de manutenção de seu modo de vida ou tem este mesmo modo de vida alterado (DAM LAM et al., 2019).

O desenvolvimento urbano e populacional, a grande demanda de energia tem trazido à tona uma rápida mudança nos padrões climáticos da Terra, afetando sobretudo as populações humanas mais vulneráveis, a grande maioria em países pobres, sobretudo nas áreas tropicais. Entretanto, os oceanos e as zonas costeiras das regiões tropicais emergem como uma fonte de soluções aos impactos ou à redução dos efeitos das mudanças climáticas, ainda que seja também vítima destas mudanças (HOEGHGULDBERG et al., 2019). 
Deste modo, entender associativamente a presença Humana nas áreas costeiras e baías tropicais é estar na vanguarda do desenvolvimento. É buscar o equilíbrio entre Humanidade e Natureza. A Revista Journal of Human and Environment of Tropical Bays se propõe a propiciar este espaço de disseminação de informação, não apenas da descrição técnica da qualidade e características ambientais, mas também, das diversas associações entre a presença Humana e seus traços culturais e comportamentais visando o EQUILÍBRIO AMBIENTAL e O DESENVOLVIMENTO SUSTENTÁVEL.

É desta forma que gostaríamos de apresentar o primeiro número da revista. Que este texto sirva como um olhar ao passado, de muitos exemplos do que fizemos acertadamente ou equivocadamente, mas sobretudo, de reestabelecermos nossa conexão com o ambiente à nossa volta.

\section{REFERÊNCIAS BIBLIOGRÁFICAS}

Barros, A. L. R. \& Rodrigues, C. G. Oliveira. DIFFERENTIATED EDUCATION AND COMMUNITY-BASED TOURISM IN THE CAIÇARA TERRITORIES OF PARATY (RJ). AMBIENTE \& SOCIEDADE, 22: E02951. 2019. HTTPS://DX.DOI.ORG/10.1590/18094422ASOC0295R1VU19L1AO

Briles, C.; Serenchenko, O.; Stevens, L.; White, A. J. \& HUONG, N. T. M. LATE HOLOCENE ANTHROPOGENIC AND CLIMATIC IMPACT ON A TROPICAL isLAND ECOSYSTEM OF NORTHERN Vietnam. Front. ECOL. EVOL. 2019. HTTPS://DOI.ORG/10.3389/FEVO.2019.00121

CENTER FOR INTERNATIONAL EARTH SCIENCE INFORMATION NETWORK - CIESIN - COLUMBIA UNIVERSITY. GRIDDED POPULATION OF THE WORLD, VERSION 4 (GP WV4): POPULATION COUNT, REVISION 11. Palisades, NY: NASA SOCIOECONomic Data and Applications Center (SEDAC). 2018. HTTPS://DOI.ORG/10.7927/H4JW8BX5

Comberti, C.; ThORNTON, T. F.; WYLlie DE ECHEVERRIA, V. \& PATTERSON, T. ECOSYSTEM SERVICES OR SERVICES TO ECOSYSTEMS? VALUING CULTIVATION AND RECIPROCAL RELATIONSHIPS BETWEEN HUMANS AND ECOSYSTEMS. GLOBAL ENVIRONMENTAL CHANGE, 34: 247-262. 2015. HTTPS://DOI.ORG/10.1016/J.GLOENVCHA.2015.07.0 $\underline{07}$.

DAM LAM, R.; GASPARATOS, A.; CHAKRABORTY, S.; Rivera, H. \& Stanley, T. Multiple Values and KNOWLEDGE INTEGRATION IN INDIGENOUS COASTAL AND MARINE SOCIAL-ECOLOGICAL SYSTEMS RESEARCH: A SYSTEMATIC REVIEW, ECOSYSTeM Services, 37: 100910.

2019,

HTTPS://DOI.ORG/10.1016/J.ECOSER.2019.100910
FAULKNER, P. LATE HOLOCENE MOLLUSC EXPLOITATION AND CHANGING NEARSHORE ENVIRONMENTS: A CASE STUDY FROM THE COASTAL MARGIN OF BLUE MUD BAY, NORTHERN AUSTRALIA, ENVIRONMENTAL ARCHAEOLOGY, 16(2): 137-150. 2011. HTTPS://DOI: $10.1179 / 174963111$ X13110803260 976

Hoegh-Guldberg. O., et al. The Ocean as a Solution to Climate Change: Five Opportunities FOR ACTION. REPORT. WASHINGTON, DC: WORLD RESOURCES INSTITUTE. 2019. AVAILABLE ONLINE AT HTTP://WWW.OCEANPANEL.ORG/CLIMATE

JONKER, G. \& HARMSEN, J. StRUCtURING the SUSTAINABILITY CONTEXT. ENGINEERING FOR SUSTAINABILITY, ED. ELSEVIER. 2012. HTTPS://DOI: 10.1016/B978-0-444-538468.00003-2

Longhurst, A. R.; Pauly, D., Ecology of Tropical Oceans. ACADEMIC PRESS, PAgeS IX-XI, 1987. HTTPS://DOI.ORG/10.1016/B978-0-12455562-4.50003-3.

Marean, C. W., Bar-MattheWs, M., Bernatchez, J., Fisher, E., Goldberg, P., Herries, A. I. R. \& WILlIAMS, H. M. EARLY HUMAN USE OF MARINE RESOURCES AND PIGMENT IN SOUTH AFRICA DURING THE Middle Pleistocene. Nature, 449(7164):905908.

(2007).

HTTPS://DOI.ORG/10.1038/NATURE06204

United nations DeVelopment Programme (UNDP). BEYOND INCOME, BEYOND AVERAGES, BEYOND TODAY: INEQUALITIES IN HUMAN DEVELOPMENT IN THE 21St CENTURY. HUMAN DEVELOPMENT REPORT 2019. DiSPONÍVEL EM http://hdr.undp.org 
SinaY, L.; SinaY, M. C. F., CARTer, R. W. (Bill) \& PASSOS, F. V. A. TRADITIONAL PEOPLE, PROTECTED AREAS AND TOURISM: A 15-YEAR BRAZILIAN CASE STUDY of Cultural Change. Ambiente \& Sociedade, 22: E00704. 2019.

HTTPS://DX.DOI.ORG/10.1590/1809-

4422ASOC0070R4VU19L1AO

SKINNER, L. F. GERENCIAMENTO AMBIENTAL MARINHO. Diversidade E GeStÃo 1(1): 145162. 2017. DISPONÍVEL EM HTTP://WWW.ITR.UFRRJ.BR/DIVERSIDADEEGESTAO/WP -CONTENT/UPLOADS/2017/07/11-GERENCIAMENTOAMBIENTAL-MARINHO LFSKINNER.PDF

TITTENSOR, D. P.; MORA, C.; JETZ, W.; LOTZE, H. K.; RiCARD, D.; VANDEN BERGHE, E. \& WORM, B. GLOBAL PATTERNS AND PREDICTORS OF MARINE BIODIVERSITY ACROSS TAXA. NATURE 466: 1098-1101. 2010. DOI: 10.1038/NATURE09329; DATA URL: HTTP://DATA.UNEP-WCMC.ORG/DATASETS/17

TORRE-CASTRO, M. DE LA \& RÖNNBÄCK, P. LINKS BETWEEN HUMANS AND SEAGRASSES-AN EXAMPLE FROM TROPICAL EAST AFRICA, OCEAN \& COASTAL MANAGEMENT, 47(7-8): 361-387, 2004. HTTPS://DOI.ORG/10.1016/J.OCECOAMAN.2004.07. $\underline{005}$.

WALter, R. C., Buffler, R. T., BRUggemanN, J. H., Guillaume, M. M. M., Berhe, S. M., Negassi, B., ... GAGNON, M. (2000). EARLY HUMAN OCCUPATION OF THE RED SEA COAST OF ERITREA DURING THE LAST interglacial. NATURE， 405(6782)， 65-69. HTTPS://DOI.ORG/10.1038/35011048 